\title{
Preface, Vorwort, Préface
}

This journal was founded in 1957 by Professor Eberhard Zwirner who, as its first chief editor, has made it into a truly international forum of phonetic discussion. He has now handed over the editorship to his former collaborators W. Bethge, H. Pilch and G. Ungeheuer. His successors are painfully aware of the great legacy which they have been called upon to administer.

'Phonetica' will remain a journal devoted to the full width of 'Phonetic Sciences' publishing contributions to instrumental phonetics, the phonemic structure of different languages and the theory of phonetics as well as book reviews. The editors reserve the right, after critical

examination, to ask authors for certain revisions of their manuscripts. They will also ask their coeditors for advice when they feel that this is appropriate. The list of co-editors has been overhauled with this end in view.

Starting with the present volume, 'Phonetica' has been designated the organ of the International Society of Phonetic Sciences. The editors profoundly appreciate this distinction which they feel is due chiefly to the services of Professor Zwirner. They are looking for the cooperation of phoneticians of all schools and all countries. Only such co-operation can make 'Phonetica' what it set out to be - a journal which truly represents the international community of phoneticians. Bonn Wolfgang Bethge

Freiburg i.Br. Herbert Pilch

Bonn Gerold Ungeheuer

Vorwort

Was die Zeitschrift «Phonetica» ist, verdankt sie ihrem Gründer und langjährigen Hauptherausgeber Eberhard Zwirner. Er hat «Phonetica» zu einer echten internationalen Zeitschrift gemacht, in der Forscher aus aller Herren Lander veröffentlichen und die in alien Ländern gelesen wird. Seine Nachfolger, W. Bethge, H. Pilch und G. Ungeheuer, haben eine Verpflichtung übernommen, die ihnen schwere Verantwortung auferlegt.

Im Sinne Eberhard Zwirners wird «Phonetica» auch weiterhin die gesamte Phonetik umfassend zu vertreten suchen, ohne sich auf besondere Fragestellungen oder bestimmte Schulen einzuengen. Die Herausgeber behalten sich vor, eingereichte Manuskripte nach gründ-licher Pruning mit der Bitte urn Umarbeitung zurückzugeben und bestimmte Änderungen vorzuschlagen. Gegebenenfalls werden sie dazu den Rat der Mitherausgeber in Anspruch nehmen. Die Mitherausgeber sind im Hinblick auf aktive Mitarbeit neu zusammengestellt worden.

Ab Band 25 wird «Phonetica» als Organ der Internationalen Gesellschaft für Phonetische Wissenschaften erscheinen.Wir danken besonders Herrn Professor Bertil Malmberg, dem Präsidenten der Gesellschaft, für das damit zum Ausdruck gebrachteVertrauen, das gewiß in erster Linie Eberhard Zwirner gilt. In diesem Sinne laden wir alle Phonetiker ein, in unserer Zeitschrift zu veröffentlichen. Nur dank ihrer dauernden Mitarbeit wird unsere Zeitschrift auch weiterhin die Internationale 
Zusammenarbeit des phonetischen Fachkollegiums verkörpern können.

Bonn Wolfgang Bethge

Freiburg i. Br. Herbert Pilch

Bonn Gerold Ungeheuer

Preface

Le professeur Eberhard Zwirner, fondateur dujournal «Phonetica» et éditeur principal des volumes 1-20, vient de Conner Гédition à ses anciens collaborateurs W. Bethge, H. Pilch et G. Ungeheuer. Sous la direction de M. Zwirner «Phonetica» est devenu un journal de rang international qui a attire la collaboration des phonéticiens dans tous les pays du monde. «Phonetica» cherchera à rester fidèle à la tradition établie par son fondateur. Elle continuera à publier des recherches dans tous les do-maines des «sciences phonétiques »-phonétique expérimentale, structure phonologique des différentes langues, théorie de la phonétique, compterendus de livres phonétiques. Les éditeurs réserveront le droit de soumettre les manuscripts à Texamen critique et de demander des changements aux auteurs. Ils s'en remetteront au conseil de leurs co-éditeurs dont ils retiendront ceux qui offrirent une collaboration active.

A partir du volume 25, «Phonetica» devient le journal de la Société Internationale des Sciences Phonétiques sous la présidence du professeur Bertil Malmberg. Nous lui savons gré d'accorder cet honneur à notre journal et à son fondateur. Nous prions les phonéticiens de tous les pays de vouloir utiliser les services de notre journal. Que «Phonetica» représente, à Tavenir aussi bien qu'au passé, la communauté Internationale des chercheurs de phonétique.

Bonn Wolfgang Bethge

Freiburg i. Br. Herbert Pilch

Bonn Gerold Ungeheuer 